\title{
Analysis of Radial Distribution Systems with FACTS Devices Using a Fast Line Flow-Based Algorithm
}

\author{
L Dinesh \\ Assistant Professor, ANITS, Sangivalasa \\ J. Uday Venkatesh \\ Assistant Professor, ANITS, Sangivalasa \\ L Vijay \\ Assistant Professor, ANITS, Sangivalasa
}

\begin{abstract}
The electrical energy produced at the generating station is conveyed to the consumers through a network of transmission and distribution systems. It is often difficult to draw a line between the transmission and distribution systems of a large power system. It is impossible to distinguish the two merely by their voltage because what was considered as a high voltage because what was considered as a high voltage a few years ago is now considered as a low voltage. In general, distribution system is that part of power system which distributes power to the consumers for utilization. Analysis of radial distribution systems with embedded series FACTS devices is facilitated by a formulation of power flow equations with bus voltage magnitudes and line flows as independent variables The line flow-based (LFB) formulation is shown to provide easy implementation with multiple series FACTS devices in the system and enable direct evaluation of the FACTS device ratings.
\end{abstract}

Keywords: FACTS, LFB, Radial distribution system

DOI: $10.7176 / \mathrm{NCS} / 10-01$

Publication date:July $31^{\text {st }} 2019$

\section{INTRODUCTION}

All distribution of electrical energy is done by constant voltage system. In practice, the following distribution circuits are generally used.In this system, separate feeders radiate from a single substation and feed the distributors at one end only. Fig 1.1 shows a single line diagram of a radial system for d.c. distribution where a feeder OC supplies a distributor AB at point A. Obviously, the distributor is fed at one end only i.e., point A is this case. Fig 1.2 shows a single line diagram of radial system for a.c. distribution.

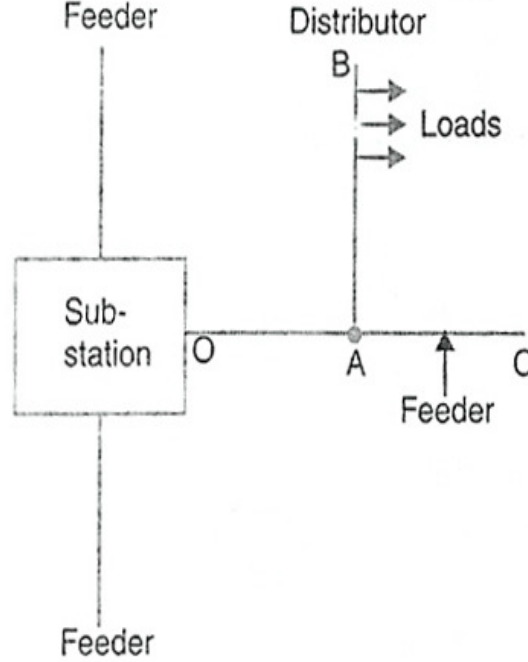

(i)

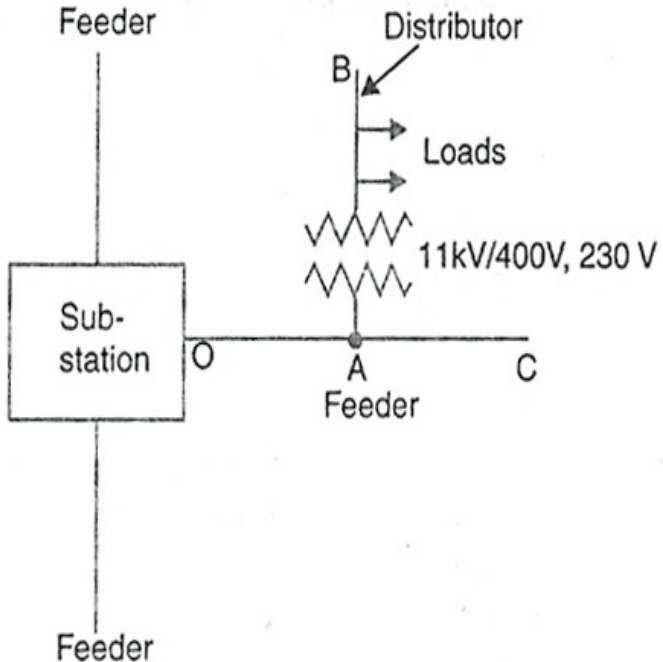

(ii)

Fig: 1.1 Radial Systems

This is the simplest distribution circuit and has the lowest initial cost. However, it suffers from the following drawbacks:

The end of the distributor nearest to the feeding point will be heavily loaded.

The consumers are dependent on a single feeder and single distributor. Therefore, any fault on the feeder or distributor cuts off supply to the consumers who are on the side of the fault away from the substation. 
The consumers at the distant end of the distributor would be subject to serious voltage fluctuations when the load on the distributor changes. Due to these limitations, this system is used for short distances only.

1.2.2. Ring Main System

In this system, the primaries of distribution transformers form a loop. The loop circuit starts from the substation bus-bars, makes a loop through the area to be served, and returns to the substation. Fig 1.2 shows the single line diagram of ring main system for a.c distribution where substation supplies to the closed feeder LMNOPQRS. The distributors are tapped from different points $\mathrm{M}, \mathrm{O}$ and $\mathrm{Q}$ of the feeder through distribution transformers.

The ring main system has the following advantages There are less voltage fluctuations at consumer's terminals. The system is very reliable as each distributor is fed via two feeders. In the event of fault on any section of the feeder, the continuity of supply is maintained. For example, suppose that fault occurs at any point F of section SLM of the feeder. Then section SLM of the feeder can be isolated for repairs and at the same time continuity of supply is maintained to all the consumers via the feeder SRQPONM.

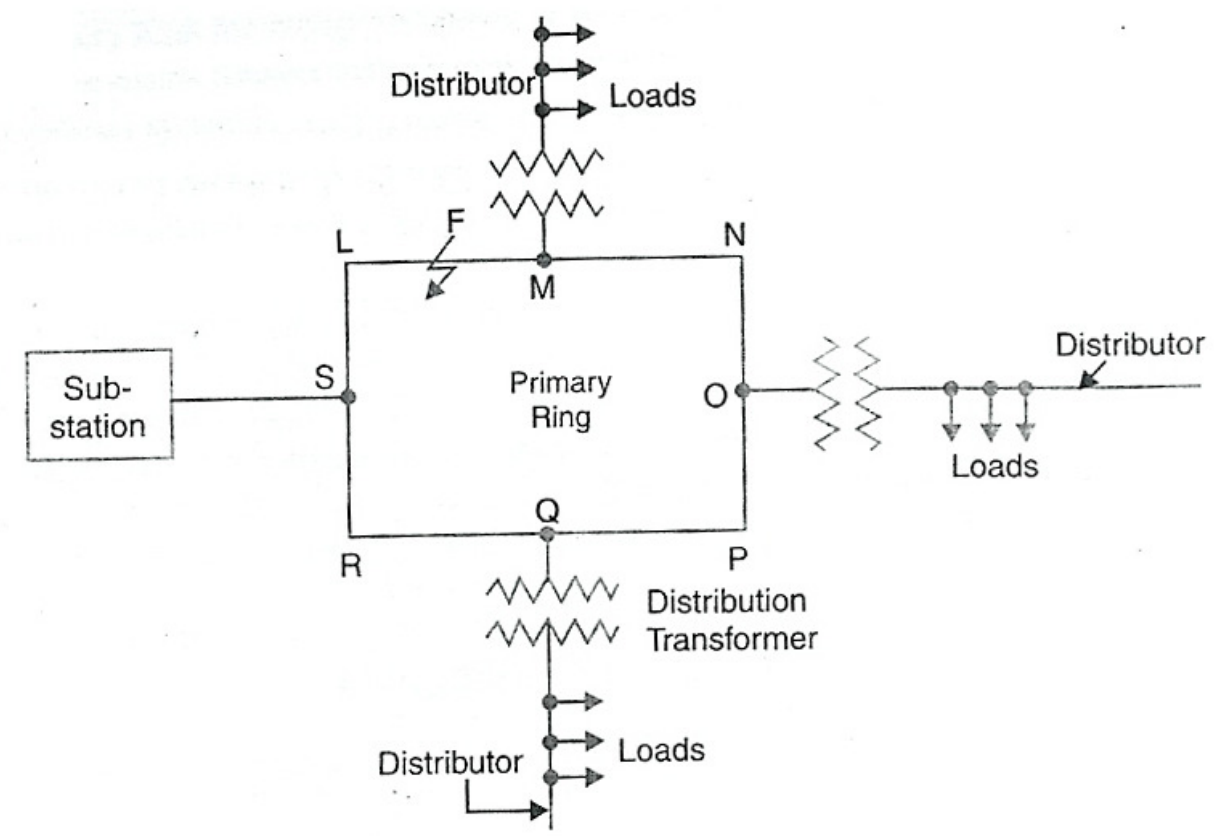

Fig 1.2 Ring main System

\subsection{Distribution Load Flows}

In this work main consideration is radial distribution system. Distribution power flow methods reported in the literature and actually implemented prefer to calculate line flows and voltage magnitudes using forward and reverse sweeps along a radial line. Mainly tree load flows are there.

1. LFB (Line Flow Based) algorithm

2. Decoupled LFB algorithm

3. Linear LFB algorithm

As stated in the introduction, there is a large incentive for the utilization of the power semiconductor technology in order to increase the network laudability. The faster response of the semiconductor-based controllers helps not only in handling dynamic problems but also in the steady-state problem of power-flow control. The main disadvantages of the mechanically switched controllers are the discrete control and the wear out of mechanical switches. The FACTS controllers provide smooth control with no (or few) mechanical parts and with high reliability.

FACTS technology opens up new opportunities for controlling power and enhancing the usable capacity of present, as well as new and upgraded, lines. The possibility that current through a line can be controlled at a reasonable cost enables a large potential of increasing the capacity of existing lines with larger conductors, and use of one of the FACTS Controllers to enable corresponding power to flow through such lines under normal and contingency conditions. By providing added flexibility, FACTS Controllers can enable a line to carry power closer to its thermal rating. Mechanical switching needs to be supplemented by rapid-response power electronics. It must be emphasized that FACTS is an enabling technology, and not a one-on-one substitute for mechanical switches. 


\section{LFB RADIAL DISTRIBUTION POWER FLOW MODEL}

The nodal variables of bus voltage magnitudes and phase angles in the common power flow models do not reflect the ultimately required practical knowledge of the line flows. The power system operation focuses on maintaining a satisfactory level of voltage magnitudes, while dispersing real power and reactive power over transmission lines or feeders to the loads. Choice of line flows as variables will provide greater flexibility in problem resolution from a practical viewpoint.

The "line flow model" equations are derived using graph theory. In a power system structure, the line segments are called elements and their terminals are called nodes (buses). From graph-theory, concepts of trees and paths are used in developing the KCL and KVL equations through incidence matrices.

Real and reactive power balance equations at all busses except slack bus can be written using the incidence matrix. Since all shunt connections are excluded in the incidence matrix, their real and reactive power contributions are accounted for separately in the power balance equations. Real and reactive power loads, shunt capacitors and line charging susceptances can be treated as shunt branches. The real and reactive power mismatch equation of each bus except the slack bus is consider.

In this chapter, basic discussion is related to LFB, Decoupled LFB and linear LFB. Fig.2.1 shows IEEE 13 node test feeder system. This 13 bus system consist of 12 lines, 1 regulator, 1 transformer and 1 switch. In practice standard system contains 3 phase unbalanced system data. This data is converted to 3 phase balanced data or 1phase data. Positive sequence of 3 phase unbalanced is nothing but a balanced domain and it simplifies the calculations.

The radial distribution system with thirteen nodes and twelve lines is shown Fig.2.1. Lines have only series impedances. The nodes are numbered arbitrarily. This typical distribution network graph is redrawn in Fig.2.2. with bus numbers in a perticular sequence starting from 1. Although bus-1 is given to the source bus here, the others encircled are arbitrary numbers as given in the original data list. This is useful when the network is reconfigured to meet the demand under different load and feeder scenarios. The flows in the branches are always oriented away from the source node, and so the direction arrows are ignored.

\subsection{Radial Distribution Network}

The radial distribution network graph has a tree structure with no loops as shown in Fig.2.2. The total number of lines equals the number of buses minus one. The pattern of incidence matrix depends on the order of lines and nodes, the incidence matrix of given distribution network is shown Fig.2.3. The Arbitrary order incidence matrix have a structure that depends on the order in which the lines are read from the data. Further, the incidence matrix is non square and singular. Any line oriented from a bus is given +1 and for towards node - 1 is assigned if the slack bus is excluded, the incidence matrix becomes square and non singular.

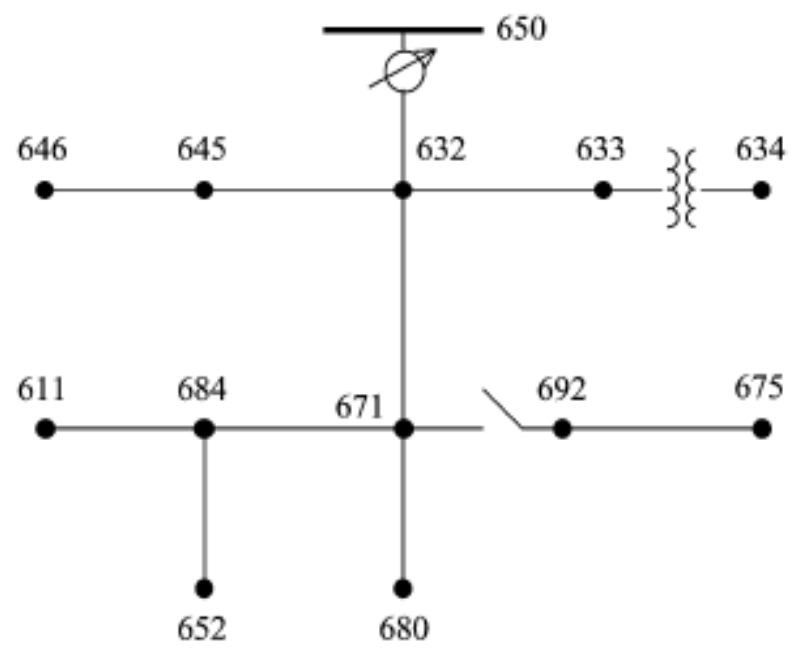

Fig.2.1 IEEE 13-node test feeder 


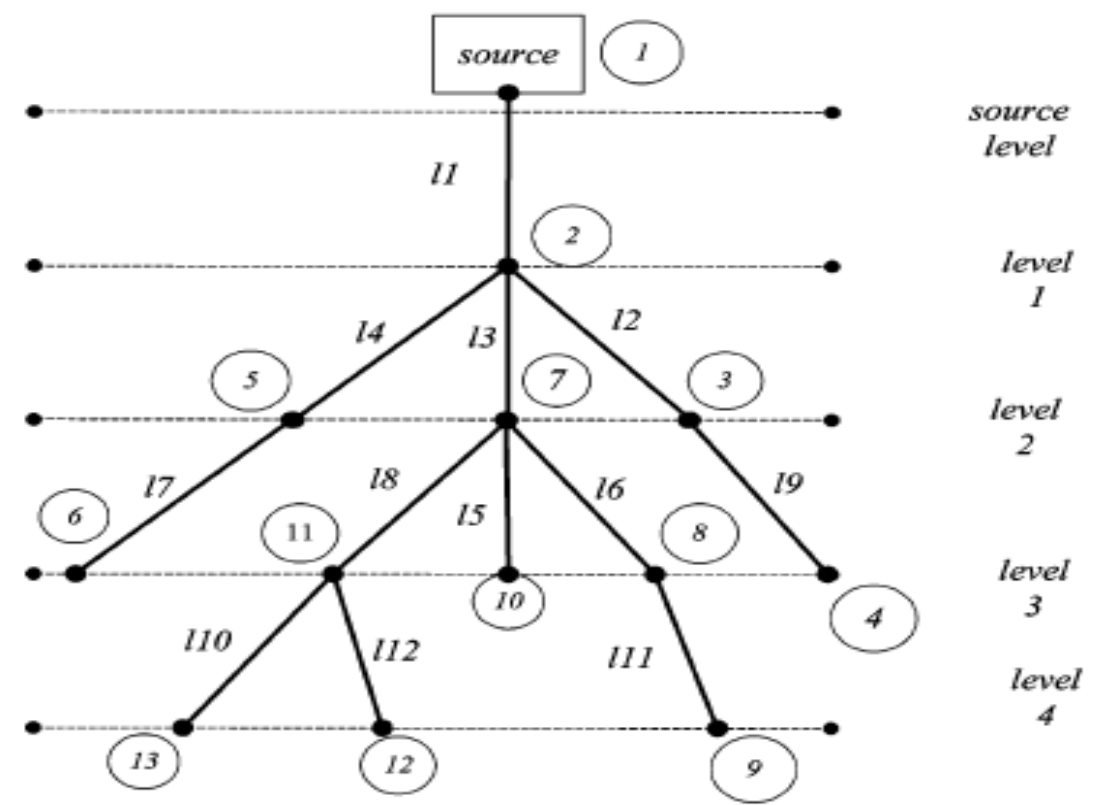

Fig.2.2: Graph of IEEE 13-node feeder

\section{BFS (BREADTH FIRST SEARCH) METHOD}

BFS is a web page searching method. BFS is a uniformed search method that aims to expand and examine all nodes of a graph systematically in search of a solution. In other words, it exhaustively searches the entire graph without considering the goal until it finds it. It does not use a heuristic.

The basic idea of BFS is to point out to as many buses as possible before penetrating deep into a tree. This means that we visit all the buses adjacent to the current level before going on to another one.

The brief description of BFS to renumber buses and branches may be summarized in the following three steps for building an optimal BFS tree.

1. Start at source bus as the first level and fan out to the "downstream" buses as the next levels.

2. On the same level, all bus numbers are ordered consecutively.

3. Branch renumbering is similar to that of the bus renumbering. At any level, a branch number is one less than the "upstream" bus number.

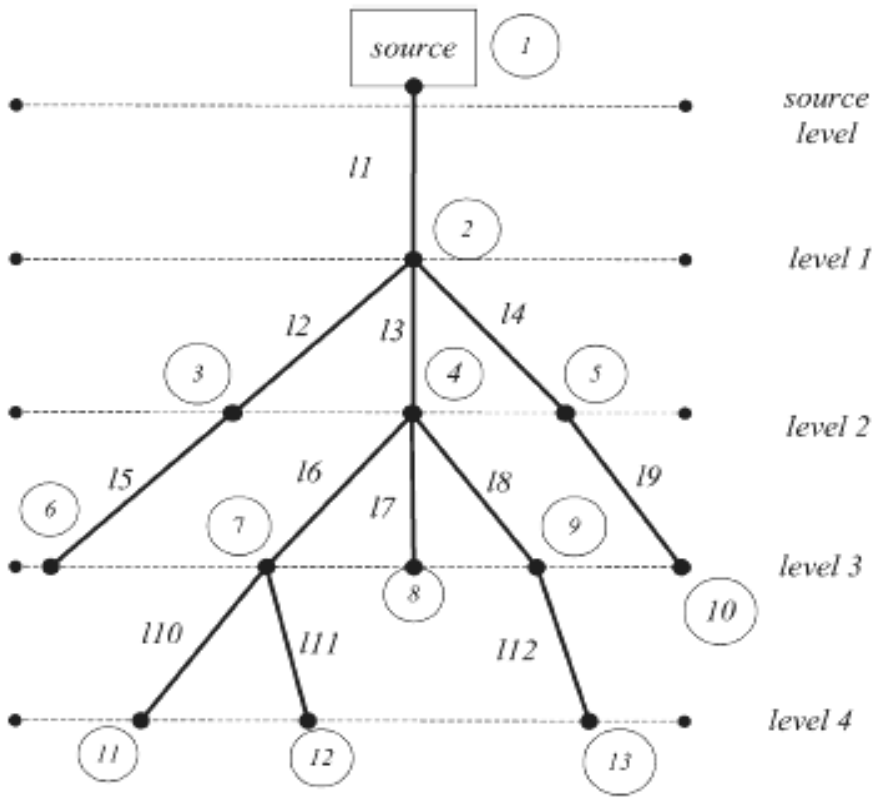

Fig.3.1: BFS numbering tree of the IEEE 13-node system. 


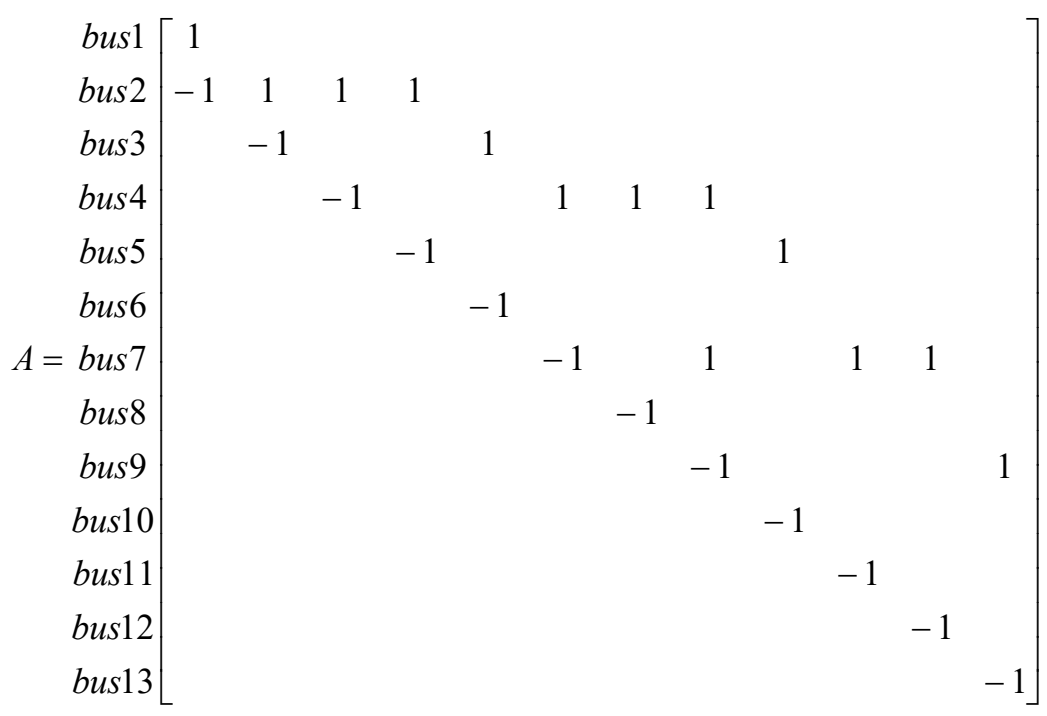

Fig.3.2: Reorder Incidence Matrix for BFS optimal tree

An example of using the BFS algorithm is illustrated using the graph of IEEE 13 node feeder Fig.2.2, including the source node (at root, originally named as 650) called Bus 1, is shown in incidence matrix Fig.2.3. To conform to LFB load flow equations of the later section, the rows of the matrix are related to buses and its columns to branches. The BFS renumbering is applied to the 13-node test feeder. Before renumbering that test system is divided different sections. The renumbering is given according to the sections. The optimal BFS tree of the IEEE 13-node feeder is shown in Fig.3.1. Its reordered incidence matrix, including the source node, in shown in Fig.3.2. After the row corresponding to the source node is deleted, bus incidence matrix $A$ becomes the upper triangle matrix in Fig.3.2. This sparse upper triangle incidence matrix results in reducing computational effort during the iterative process.

\section{IEEE 34 BUS SYSTEM RESULTS}

\subsection{Decoupled LFB Method Results}

Table 4.1.Line Data of load $\mathrm{Pl}$ and $\mathrm{Q} 1$ at end buses of each line

\begin{tabular}{|l|l|l|l|l|l|l|}
\hline LINE NO & LP & LQ & R & X & P1 & Q1 \\
\hline 1 & 1 & 2 & 0.5473 & 0.4072 & 0 & 0 \\
\hline 2 & 2 & 3 & 0.3669 & 0.273 & 0.0055 & 0.0029 \\
\hline 3 & 3 & 4 & 6.835 & 5.086 & 0.0055 & 0.0029 \\
\hline 4 & 4 & 5 & 1.0253 & 0.544 & 0.0016 & 0.0008 \\
\hline 5 & 4 & 6 & 7.953 & 5.917 & 0.0016 & 0.0008 \\
\hline 6 & 6 & 7 & 6.305 & 4.6913 & 0 & 0 \\
\hline 7 & 7 & 8 & 0.0032 & 0.0016 & 0 & 0 \\
\hline 8 & 8 & 9 & 0.0992 & 0.0494 & 0 & 0 \\
\hline 9 & 9 & 10 & 0.302 & 0.1603 & 0.0005 & 0.0002 \\
\hline 10 & 9 & 11 & 3.267 & 1.626 & 0.0034 & 0.0017 \\
\hline 11 & 10 & 12 & 8.506 & 4.515 & 0.0049 & 0.0024 \\
\hline 12 & 11 & 13 & 0.5352 & 0.2841 & 0.0169 & 0.0087 \\
\hline 13 & 11 & 14 & 0.2688 & 0.1338 & 0.004 & 0.002 \\
\hline 14 & 12 & 15 & 2.427 & 1.2883 & 0.0011 & 0.0005 \\
\hline 15 & 14 & 16 & 6.5404 & 3.255 & 0.0135 & 0.007 \\
\hline 16 & 16 & 17 & 0.1664 & 0.0828 & 0.0067 & 0.0033 \\
\hline 17 & 17 & 18 & 4.1213 & 2.1874 & 0.0004 & 0.0002 \\
\hline 18 & 17 & 19 & 11.785 & 5.865 & 0.0004 & 0.0002 \\
\hline 19 & 19 & 20 & 0.0032 & 0.0016 & 0 & 0 \\
\hline 20 & 20 & 21 & 1.5679 & 0.7802 & 0.0015 & 0.0007 \\
\hline 21 & 20 & 22 & 0 & 0 & 0.0049 & 0.0025 \\
\hline 22 & 21 & 23 & 0.2862 & 0.152 & 0 & 0 \\
\hline 23 & 21 & 24 & 1.8655 & 0.9283 & 0.0032 & 0.0017 \\
\hline 24 & 22 & 25 & 2.24 & 1.666 & 0.0178 & 0.009 \\
\hline 25 & 24 & 26 & 0.6464 & 0.3217 & 0.09 & 0.045 \\
\hline & & & & & &
\end{tabular}




\begin{tabular}{|l|l|l|l|l|l|l|}
\hline LINE NO & LP & LQ & R & X & Pl & Ql \\
\hline 26 & 24 & 27 & 0.0896 & 0.0446 & 0.0348 & 0.0212 \\
\hline 27 & 26 & 28 & 0.8576 & 0.427 & 0.0009 & 0.0005 \\
\hline 28 & 27 & 29 & 0.432 & 0.215 & 0.0122 & 0.0063 \\
\hline 29 & 28 & 30 & 0.2752 & 0.1369 & 0.0864 & 0.1258 \\
\hline 30 & 28 & 31 & 0.0896 & 0.0446 & 0.0094 & 0.0062 \\
\hline 31 & 29 & 32 & 1.165 & 0.5796 & 0.0028 & 0.0014 \\
\hline 32 & 31 & 33 & 0.5894 & 0.436 & 0.0068 & 0.0034 \\
\hline 33 & 32 & 34 & 0.1696 & 0.0844 & 0.0028 & 0.0014 \\
\hline
\end{tabular}

Table 4.2.Real \&Reactive Power at each Bus

\begin{tabular}{|c|c|c|}
\hline BUS NO & Peff & Qeff \\
\hline 1 & 0.3538 & 0.3594 \\
\hline 2 & 0.3538 & 0.3594 \\
\hline 3 & 0.3483 & 0.3565 \\
\hline 4 & 0.3428 & 0.3536 \\
\hline 5 & 0.0016 & 0.0008 \\
\hline 6 & 0.3396 & 0.352 \\
\hline 7 & 0.3396 & 0.352 \\
\hline 8 & 0.3396 & 0.352 \\
\hline 9 & 0.3396 & 0.352 \\
\hline 10 & 0.0338 & 0.0174 \\
\hline 11 & 0.3053 & 0.3344 \\
\hline 12 & 0.0304 & 0.0157 \\
\hline 13 & 0.004 & 0.002 \\
\hline 14 & 0.2964 & 0.33 \\
\hline 15 & 0.0135 & 0.007 \\
\hline 16 & 0.2953 & 0.3295 \\
\hline 17 & 0.2886 & 0.3262 \\
\hline 18 & 0.0004 & 0.0002 \\
\hline 19 & 0.2878 & 0.3258 \\
\hline 20 & 0.2878 & 0.3258 \\
\hline 21 & 0.1963 & 0.2801 \\
\hline 22 & 0.09 & 0.045 \\
\hline 23 & 0.0032 & 0.0017 \\
\hline 24 & 0.1882 & 0.2759 \\
\hline 25 & 0.09 & 0.045 \\
\hline 26 & 0.062 & 0.0365 \\
\hline 27 & 0.1084 & 0.2304 \\
\hline 28 & 0.0272 & 0.0153 \\
\hline 29 & 0.1075 & 0.2299 \\
\hline 30 & 0.0094 & 0.0062 \\
\hline 31 & 0.0056 & 0.0028 \\
\hline 32 & 0.0211 & 0.1041 \\
\hline 33 & 0.0028 & 0.0014 \\
\hline 34 & 0.0143 & 0.1007 \\
\hline
\end{tabular}

\begin{tabular}{|l|l|l|}
\hline \multicolumn{1}{|c|}{28} & 0.9365 & 2.7018 \\
\hline 29 & 0.9364 & 2.7248 \\
\hline 30 & 0.9365 & 2.7019 \\
\hline 31 & 0.9365 & 2.7018 \\
\hline 32 & 0.936 & 2.7536 \\
\hline 33 & 0.9365 & 2.7017 \\
\hline 34 & 0.936 & 2.7577 \\
\hline
\end{tabular}




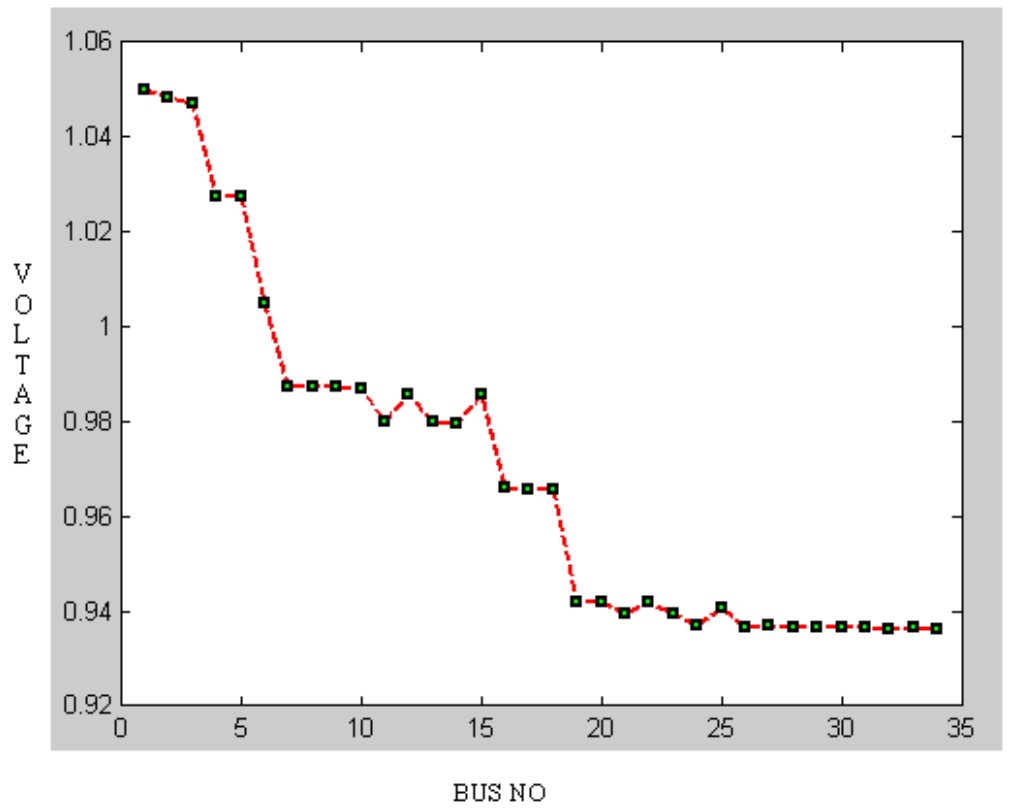

Fig 4.1.Graph: Busno $V$ s Voltage

LFB and D-LFB methods have converged in 4 iterations for 13 and 34 bus systems. The linear LFB has taken only two iterations but the results are not accurate enough.

Table 4.3. Comparison of solution times, in seconds.

\begin{tabular}{|c|c|c|c|}
\hline IEEE Distribution systems & LFB & D-LFB & Linear LFB \\
\hline IEEE 13-node & 0.03 & 0.02 & 0.01 \\
\hline IEEE 34-node & 0.04 & 0.03 & 0.02 \\
\hline
\end{tabular}

It may be noted that with good accuracy and reduced time the D-LFB establishes its superiority other two methods (LFB and linear-LFB).

\section{CONCLUSIONS}

In this thesis three algorithm for line flow study of radial distribution system have been considered. Three algorithms Line Flow Based (LFB), Decoupled LFB, Linear LFB formulation and Decoupled LFB with embedded series FACTS devices (TCSC and TCVR) are implemented in this thesis and tested on IEEE 13- bus and IEEE 34-bus systems.

Breadth first search method is used for network modification. The incidence matrix was converted to upper triangular matrix. This method incorporated with LFB, it is termed Decoupled LFB. In this method, commutation time is low compared to LFB.

By neglecting losses in the formation of LFB method a new method called linear LFB was developed.

The three algorithms LFB, Decoupled LFB, Linear LFB are implemented on IEEE 13 bus and IEEE 34 bus distribution systems and observed that Decoupled LFB is taking less time and produced accurate results compared other two algorithms. Among three methods the Decoupled LFB is the best one. Thus, in this thesis Decoupled LFB embedded with series FACTS devices is implemented and developed a software package. Variable swapping method is incorporated in the Decoupled LFB for the system with TCSC or TCVR.

\section{References}

[1] P. Yan and A. Sekar, "Analysis of radial distribution systems with embedded series facts devices using a fast line flow-based algorithm,"IEEE Transactions on Power Systems, vol. 20, no. 4, pp. 1775-1782, 2005.

[2] W. F. Tinney and C. Z. Hart, "Power flowsolution by Newton's method,"IEEE Trans. Power App. Syst., vol. 86, no. 11, pp. 1449-1456, Nov.1967.

[3] B. Stott and O. Alsac, "Fast decoupled load flow," IEEE Trans. PowerApp. Syst., vol. 93, no. 3, pp. 859-869, May/Jun. 1974.

[4] N. G. Hingorani and L. Gyugyi, Understanding FACTS: Concept and Technology of Flexible AC Transmission Systems. New York: IEEE Press, 1999.

[5] N. M. Peterson and W. Meyer, "Automatic adjustment of transformer and phase-shifter taps in the Newton power flow," IEEE Trans. Power App. Syst., vol. PAS-90, no. 3, pp. 103-108, Jan./Feb. 1971.

[6] C. R. Fuerte-Esquivel and E. Acha, "Newton-Raphson algorithm for the reliable solution of large power networks with embedded FACTS devices," in Proc. Inst. Elect. Eng., Gener., Transm., Distrib., vol. 143, 
Sep. 1996, pp. 447-454.

[7] , "A Newton-type algorithm for the control of power flow in electrical power networks," IEEE Trans. Power Syst., vol. 12, no. 4, pp. 1474-1480, Nov. 1997.

[8] M. R. Salem, L. A. Talat, and H. M. Soliman, "Voltage control by tapchanging transformers for a radial distribution network," in Proc. Inst. Elect. Eng., Gener., Transm., Distrib., vol. 144, Nov. 1997, pp. 517520.

[9] P. A. N. Garcia, J. L. R. Pereira, and S. Carneiro, "Voltage control devices models for distribution power flow analysis," IEEE Trans. Power Syst., vol. 16, no. 4, pp. 586-593, Nov. 2001.

[10] C. Trevino, "Cases of difficult convergence in load flowstudies," in Proc. IEEE Paper 71-62-PWR, Summer Meeting, Los Angeles, 1970.

[11] W. H. Kersting, Distribution System Modeling and Analysis. Boca Raton, FL: CRC, 2002.

[12] DEWorkstation, Electric Power Research Institute, Palo Alto, CA.

[13] G. X. Luo and A. Semlyen, "Efficient load flow for large weakly meshed networks," IEEE Trans. Power Syst., vol. 5, no. 4, pp. 1309-1316, Nov. 1990.

[14] S. Cheng and D. Shirmohammadi, "A three phase power flow method for real time distribution system analysis,” IEEE Trans. Power Syst., vol. 10, no. 2, pp. 671-679, May 1995.

[15] M. E. Baran and F. F. Fu, “Optimal sizing of capacitors placed on a radial distribution system," IEEE Trans. Power Del., vol. 4, no. 1, pp. 735-742, Jan. 1989.

[16] A. G. Exposito and E. R. Ramos, "Reliable load flowtechnique for radial distribution networks," in Proc. IEEE PE-344-PWRS-0-12, 1996.

[17] J. Gotham and G. T. Geydt, "FACTS device models for power studies," in Proc. 27th NAPS, Bozeman, MT, Oct. 2-3, 1995, pp. 514-519.

[18] L. Gyugyi, T. R. Rietman, S. L.Willians, T. R. Rietman, D. R. Torgerson, and A. Edris, "The unified power flow controller: A new approach to power transmission control,” IEEE Trans. Power Del., vol. 10, no. 2, pp. 1085-1097, Apr. 1995.

[19] G. W. Stagg and A. H. El_Abiad, Computer Methods for Power System Analysis. New York: McGrawHill, 1971.

[20] J. R. Patel, "Power flow analysis using modified tableau formulation of circuit equations," Master's thesis, Tennessee Technological Univ., Cookeville, TN, 1992.

[21] F. Zhang and C. S. Cheng, "A modified newton method for radial distribution system power flow analysis," IEEE Trans. Power Syst., vol. 12, no. 1, pp. 389-397, Feb. 1997.

[22] R. J. Wilson and J. J. Watkins, Graphs: An Introductory Approach. New York: Wiley, 1990.

[23] W. M. Kersting and L. Willis, "Radial distribution test systems," IEEE Trans. Power Syst., vol. 6, no. 3, pp. 975-985, Aug. 1991.

[24] IEEE Distribution Feeder Test Result. IEEE Distribution System Analysis Subcommittee. [Online]. Available: http//ewh.ieee.org/soc/pes/dsacom/testfeeders.html

[25] M. Najork and J. L. Wiener, "Breadth-first search crawling yields high quality pages," in Proc. ACM 1-58 113-348-0/01/0005, WWW10, Hong Kong, May 2-5, 2001. 